\title{
EUCALYPTUS SPECIES SELECTION FOR SOIL CONSERVATION IN SEASONALLY DRY HILL COUNTRY - TWELFTH YEAR ASSESSMENT
}

\author{
B.T. BULLOCH \\ DSIR Fruit and Trees, Private Bag, Palmerston North, New Zealand
}

(Received for publication 9 January 1992)

\begin{abstract}
Hillside stabilisation tree plantings are aimed at maintaining erosion-prone land in pastoral use. Species selection trials on drought-prone hillsides in the Wairarapa district (east coast, lower North Island, New Zealand) included over 120 provenances of eucalypts drawn from over 60 species. The trial sites of Pakaraka and Kahuiti, planted in 1979, were re-assessed in 1991 after an earlier comprehensive measurement in 1984. On the basis of the latest data, larger-growing eucalypts suited to hillside plantings in this district are Eucalyptus regnans F. Muell., E. obliqua L'Herit, E. fastigata Deane et Maid., E. botryoides Sm., E. botryoides $\times$ saligna Sm., E. nitens (Deane et Maid.) Maid., E. fraxinoides Deane et Maid., E. sieberi L. Johnson, E. delegatensis R.T. Bak., E. viminalis Labill, $E$. globoidea Blakely, E. smithii R.T.Bak., and E. oreades R.T. Bak. Suitable smaller stature species are E. amygdalina Labill., E. cordata Labill., E. brookeriana A.M.Gray, E. radiata Sieb.ex DC., E. cladocalyx F. Muell., E. pulchella Desf., E. agglomerata Maid., and E. nitida Hook. f. The larger trees would serve best in conservation/production/agroforestry regimes, the smaller at wider spacings in conservation/pastoralism regimes. Provenance comparisons within species generally support the findings of more extensive genetic improvement trials focused on the eucalypts with most potential for timber production.
\end{abstract}

Keywords: soil conservation; species selection; pastoral hill country; Eucalyptus spp.

\section{INTRODUCTION}

Much of the New Zealand hill country which was formerly forested and has been cleared for pastoral farming is prone to erosion. In the Land Resource Inventory (Eyles 1983), 30\% of the North Island was mapped in units affected by soil slip erosion (defined as rapid sliding or flowing movements usually less than $1 \mathrm{~m}$ deep) and $22 \%$ in units affected by other forms of mass movement, most slopes steeper than $15^{\circ}$ being susceptible. To stabilise the land and retain it in pastoral use, an established practice is to plant trees at wide spacings on hill slopes (Hathaway 1986a) and more closely along water courses and in eroding gullies (Hathaway 1986b). Poplars and willows are widely used for these purposes, and have the particular 
advantage that they may be established from poles in the presence of grazing sheep. However, poplars and willows are insufficiently drought tolerant for drier parts of the country and for upper hillslopes in seasonally drought-prone districts.

Trees of the more drought-tolerant Eucalyptus genus have been planted for many utilitarian purposes, including soil conservation and shelter, on a wide range of sites. A number have proved well adapted to New Zealand environments (Hathaway \& Sheppard 1986). Impediments to their more intensive use have included lack of systematic evaluation, unreliable information about suitability of species and provenances, lack of quality seed supply, unreliable information on propagation and establishment techniques, and often unfavourable reputations founded on inappropriate choice of species and management techniques. During 1973-79 the Forest Research Institute initiated genetic improvement programmes for the seven species considered to have most potential for wood production (Wilcox 1980). These were E. botryoides, E. saligna Sm., E. regnans, E. delegatensis, E. fastigata, E. obliqua, and E. nitens. A further 24 species were included in species selection trials and 13 species in mountainland revegetation trials (Wilcox 1980).

The vigour and early growth rates of eucalypts help compensate for their having to be planted out in soil conservation plantings as seedlings rather than poles. Since the early 1970 s eucalypts have featured in National Plant Material Centre (later Soil Conservation Centre) trials to select trees for soil conservation and shelter in drier and drought-prone areas, especially of the South Island. A major impetus to eucalypt selection trials in the North Island was provided by the exceptionally wet winter and early spring of 1977 which resulted in extensive damage to Wairarapa hill country pastures. In conjunction with the Wairarapa Catchment Board, species selection trials were established on several hill country sites east of Masterton, the largest being at Pakaraka and Kahuiti planted in 1979. These trials included over 120 provenances of eucalypts drawn from over 60 species, as well as species of Acacia and Casuarina, clones of poplar, and Pinus radiata D. Don. For eucalypts an objective was to compare a wide range of species in systematic trials, including species of potentially smaller stature than conventional "timber eucalypts". For those eucalypts with an established reputation, an objective was a systematic comparison of provenances.

A comprehensive analysis of eucalypt data from Pakaraka and Kahuiti 5 years after planting was given by Hathaway \& King (1986). Observations included measurements of height, diameter at $1.4 \mathrm{~m}(\mathrm{dbh})$, and percentage survival; estimates of resistance to Paropsis charybdis Stål, to leafroller caterpillar, and to wind damage; and ratings of tree form including stem straightness, crown width, crown density, and branch size. Selection criteria were basically for drought-tolerant trees, an ability to establish quickly and grow out of the reach of livestock, tolerance of the variability of hill country soils, resistance to wind damage and to debilitating infestations of pests or diseases, and an ultimate form compatible with pastoral production.

Eucalyptus cordata, E. fastigata, E. fraxinoides, E. obliqua, E. pulchella, and E. regnans were ranked highly at both sites by Hathaway \& King (1986) for most of the traits assessed, and were considered the most suitable of the species tested. Those authors acknowledged, however, that larger-growing species such as E. fastigata, E. fraxinoides, E. obliqua, and $E$. regnans were likely to develop into large spreading trees that could destabilise steep slopes. Trees of ultimately smaller stature, such as E. cordata or E. pulchella, could be preferable options for steeper and upper slopes. From 1980 over 20 further eucalypt selection 
trials were established in Wairarapa, Hawke's Bay, Poverty Bay, and Northland. These were to test in other districts the better selections from the Wairarapa trials and to evaluate provenances of the peppermint eucalypts (E. amygdalina, E. nitida, and E. pulchella).

By 1991, 12 years after planting and 7 years after the assessment by Hathaway \& King, a further comprehensive assessment of the Pakaraka and Kahuiti trials was timely.

\section{METHOD \\ Sites}

The Pakaraka trial is located $8 \mathrm{~km}$ south-east of Masterton. The site consists of a hillside of shallow loess deposits over siltstone (Pirinoa hill soils and Taueru silt loam). Aspect is northerly, slope $21-25^{\circ}$, and altitude $150-250 \mathrm{~m}$. The highest part of the site is the crest of the hill. During the winter of 1977 most of the site was extensively damaged with shallow soil slips.

The Kahuiti trial site is located $28 \mathrm{~km}$ east of Masterton. The site consists of a scarp of silt and clay loam over mudstone (Taihape steepland soils). Aspect is north-westerly, slope $26-35^{\circ}$, and altitude $150-180 \mathrm{~m}$. The highest part of the site is the rim of a terrace and so the slope receives not only incident rainfall but also runoff and seepage from the portion of the catchment above. Several streamlets flow down the slope for most of the winter and ephemeral streamlets are numerous during rainfall. Earthflow and soil slip are chronic and patches of rushes indicate seepage areas low on the slope. Large areas of subsoil were exposed during and after 1977.

\section{Climate}

Meteorological records from Waingawa (south of Masterton and $8 \mathrm{~km}$ west of Pakaraka), Te Ore Ore (closer to the hills and $4 \mathrm{~km}$ north of Pakaraka), and Ngaumu Forest (9 km south of Kahuiti) indicate an average annual rainfall for the vicinity approximating $1000 \mathrm{~mm}$, the 10-90 percentile range approximating $800-1200 \mathrm{~mm}$. Pakaraka probably receives about $50 \mathrm{~mm}$ less, being located on the flank of the eastern chain of hills, and Kahuiti a similar amount more as rainfall rises towards the crest of the chain. Summer afternoons are relatively warm and winter nights relatively $\mathrm{cool}^{*}$, with sudden fluctuations in temperature common (Thompson 1982). There are about 90 days with ground frost annually at Waingawa, occasionally of the order of $-10^{\circ} \mathrm{C}$, and over 100 days at Ngaumu, but Hathaway \& King inferred that the two trial sites are largely drained of frost as none of the seedlings suffered frost damage after planting. There are about 2000 hours of sunshine annually. The district is fairly windy year round, mean daily wind run at Waingawa being $213 \mathrm{~km}$. North-easterly winds predominate in spring and summer and are often föhn-like as a result of gusty airflow over the axial ranges to the west. Average annual evapotranspiration is calculated at about $800 \mathrm{~mm}$, with annual water deficiency (wilting point deficit) of 180-270 mm for soils of low to average water-holding capacity, the deficiency being most pronounced in January and February (Meteorological Service 1986).

* Mean daily temperature at Waingawa $\left(114 \mathrm{~m}\right.$ a.s.l.) is $12^{\circ} \mathrm{C}$, daily range $11^{\circ} \mathrm{C}$, average annual maximum $31^{\circ} \mathrm{C}$, and average annual (screen) minimum $-4^{\circ} \mathrm{C}$. 


\section{Original Planting}

The trial seedlings were grown in $8 \times 8-\mathrm{cm}$ peat pots and were planted out in June about 6 months after sowing. The sites were grazed hard up to 2 weeks before planting, and then grazing stock were excluded. Wire netting was fixed to boundary fences to exclude rabbits and hares. Planting spots were sprayed with paraquat (1.2 kg a.i./ha) and simazine $(1.5 \mathrm{~kg}$ a.i./ha) a week prior to planting, and at planting $20 \mathrm{~g} \mathrm{Magamp}^{\circledR}$ (7N:14P:5K:13Mg) was incorporated into the soil around each plant. Areas where the topsoil had obviously been stripped down to the subsoil were not planted as part of the species selection trial, but some were concurrently and later used for fertiliser application trials.

Plants were spaced 3-4 m apart in rows of four, the rows being at least $4 \mathrm{~m}$ apart. Individual species were represented by plots of two rows, and provenances by single-row plots nested together with the others of that species. There were five randomised blocks at each site. (The fifth block at Kahuiti included seepage areas low on the slope, unfavourable to eucalypt survival and growth, and did not contain the complete range of provenances, and so was not included as a replication by Hathaway \& King.) During the first year the plants were released with Caragard ${ }^{\circledR}$ (active ingredients $25 \%$ terbuthylazine $+25 \%$ terbumeton).

\section{Subsequent Treatment}

Grazing stock were readmitted to the trial sites in 1983, and the Pakaraka site has continued to be regularly grazed mainly by sheep. Very little further soil slip erosion has occurred at Pakaraka since planting. Kahuiti has been grazed intermittently with cattle but is now again retired from grazing. Trial and erosion control plantings have continued on adjacent areas of the Kahuiti scarp face. Both soil slip and earth flow erosion continued after planting at Kahuiti, with consequent loss of a number of plots in the original trial.

In 1987 Pakaraka was thinned to an average of the best two trees per plot. This was intended to reduce the original density of 600-800 trees/ha to a range of 100-300 trees/ha, depending on whether plots were four plants or eight plants. In 1991 many plots had no survivors, some retained only the best one or two, and some more. Thus over much of the site the trees are still sufficiently free-standing to provide a reasonable indication of performance at "wide" spacings (conventionally 25-100 trees/ha for soil conservation hillside stabilisation plantings), but the more vigorous species, especially those in groups of nested plots, have formed closed stands despite thinning.

Kahuiti has not been thinned but survival has been lower (66\% in 1984 v. $81 \%$ at Pakaraka), a result of erosion and unfavourable siting. Fresh slips and patches of rushes which were unplanted from the outset. Overall densities at the two sites are now similar but areas of low tree density are more extensive at Kahuiti.

\section{Assessment}

In 1991 (February for Pakaraka and May for Kahuiti) the dbh of all surviving trees was measured, excluding those which were coppice regrowth. A single mean height was recorded for uncoppiced survivors in each plot. Plots were rated subjectively for vigour, resistance to damage by insects, stem form, crown form, branch form, and utility as soil conservation trees, 0-5 scales of increasing desirability being used. 
Dbh was used as the prime objective measure of tree performance (in contrast to height which was used by Hathaway \& King). Hathaway's \& King's separate ratings of damage by the eucalyptus tortoise beetle (Paropsis charybdis) and the tortricid leafroller caterpillar (Strepsicrates macropetana Meyrick) were combined in a single insect damage rating. The introduction and release of effective parasites for the eucalyptus tortoise beetle mean that this pest no longer needs to be accorded the status of a special problem (Forest Research Institute 1990). For Acacia species, resistance to Uromycladium rusts was included in the "insect" resistance rating.

The ratings of stem form, crown form, and branch form were intended to select for trees with straight trunks (or slightly wavy trunks but good single leader dominance), relatively narrow crowns in relation to height (to minimise shading of pasture), and light side branches (less likely to be prone to damage by wind). The crown density rating of Hathaway \& King, implying more dense crowns were a desirable trait, was not used. The utility rating endeavoured to assign a value to the perceived usefulness of the species for soil conservation on the type of pastoral hill country typified by the site. The trees were not rated for wind damage, as with one exception (E. pulchella at Pakaraka) little damage was evident.

Analysis of variance was applied to the data separately for the two sites. In a further departure from the presentation of Hathaway \& King, five species of Acacia and four of Casuarina were included in the data. In the analysis of species data, single mean values were used for species represented by more than one provenance. Absence of a species from one replicate at a site was treated as a missing value, but if absent from two or more replicates at a site the species was excluded from that site's analyses. An exception was survival at Kahuiti where means were also calculated, treating plots with no survivors as $0 \%$. Survival at Pakaraka was confounded by thinning and was not analysed. In the tables of results, means are not given for species not represented by four or more plants at one site. Only E. gunnii Hook. f. failed to attain this criterion at both sites. At Pakaraka, dbh was analysed both for best trees per plot and for plot means, as either data set was liable to bias where there were marked disparities of size amongst survivors in the same plots.

Species included in the analyses of variance were assigned to upper, middle, and lower performance groupings for each site. For Pakaraka these were based on rankings for $\mathrm{dbh}$ and the combined subjective ratings. For Kahuiti they were based on rankings for dbh, the combined subjective ratings, and a combination of both sets of survival means. Provenances within species in the upper grouping for each site were compared by analyses of variance using separate pooled error mean squares for ash group, peppermint group, and gum group species. Again, provenances missing from one replicate at a site were treated as missing values, and those missing from more than one were excluded from the analyses.

\section{RESULTS AND DISCUSSION \\ Species}

Species data means for Pakaraka are given in Table 1 and for Kahuiti in Table 2. Predictably, considering the range of species involved, the species effect was very highly significant for all the parameters considered. Overall means were consistently higher at Pakaraka than those of Kahuiti, by $44 \%$ for mean dbh (acknowledging that the mean dbh at 
Pakaraka has been distorted by culling), by $25 \%$ for height, and $10-80 \%$ for the subjective ratings. The replication effect was also significant for mean $\mathrm{dbh}$, height, vigour, and the utility rating at Pakaraka, all essentially decreasing with increasing height and exposure up the slope; and for survival, stem form, and branch form at Kahuiti, where actual (non-missing value) survival for the replicate most affected by post-planting soil movement was $53 \%$ and for the lowest replicate located on the seepage areas $37 \%$, both these differing from the other replicates and each other at the 5\% significance level. Whilst the differences were comparatively small, stem form and branch form ratings were highest in the $53 \%$ survival replicate and may have been an artefact of differential survival there.

Half the species at Pakaraka had mean scores of 4.0 or better for vigour. A third scored 4.0 or better for resistance to damage from insects. These two parameters were not independent, for while insect predation obviously depressed vigour for some species, vigorous regrowth also often masked the damage from previous infestations. For example, even though most $E$. nitens were carrying their $P$. charybdis-susceptible adult foliage, the mean insect resistance rating for the species was 3.0 (= "about average") and despite insect predation its mean vigour score was 4.8 . Insect predation is probably minimised by the very wide range of species (and susceptibilities) in these trials - insect population dynamics and predation could be different in a monoculture. About two-thirds of the insect damage seen was caused by $P$. charybdis and most of the rest by tortricid leafroller caterpillars. The eucalyptus leaf mining sawfly, Phylacteophaga froggatti Riek, was not seen on the sites. With the lower scores for vigour at Kahuiti (only a third of the species attaining means of 3.0 or better), insect resistance scores were similarly reduced. Ratings at Kahuiti may also have been influenced by assessment later in the growing season (May for Kahuiti, February for Pakaraka).

Corresponding site means from the 1984 assessment were compared with those of 1991 in Table 3. Overall means had increased fourfold, while heights had little more than doubled. Overall mean survival at Kahuiti had apparently changed little, remaining the same on a missing plot basis and reducing from $66 \%$ to $63 \%$ on an actual percentage basis for the four replications assessed by Hathaway \& King in 1984 (although this does represent loss of a further 70 plants in 7 years). In 1984 overall mean subjective ratings were generally higher at Kahuiti but, as noted above, this relationship was reversed in 1991. The combined insect resistance rating means of 1991 were lower than the separate Paropsis charybdis/leafroller means of 1984, as would be expected of combined values, but the difference at Pakaraka was not great and suggests less damage from insects than may have been anticipated there. The growth habit ratings were consistently higher in 1991 at both sites, the differences being larger at Pakaraka. This is likely to be an effect of the tendency towards closed stands apparently improving tree form. A degree of observer bias is also probable between the two assessments.

The performance groupings from the 1991 assessment are shown in Table 4 where the species are listed in a combined ranking for both sites. Essentially, the upper groupings succeeded on these sites according to the evaluation criteria used, the lower grouping failed, and the middle grouping performed equivocally, with some good features but most others unfavourable. Unranked species are essentially those on which judgement is being reserved because they were poorly represented, but this was mostly a reflection of poor survival. The upper grouping at Pakaraka comprised 22 species, one of which (Acacia dealbata Link.) is 
TABLE 1-Assessment of Eucalyptus, Acacia, and Casuarina species in the Pakaraka trial, 12 years after planting

\begin{tabular}{|c|c|c|c|c|c|c|c|c|c|c|c|c|}
\hline Species & $\begin{array}{l}\text { No. of } \\
\text { provenances }\end{array}$ & $\begin{array}{l}\text { Best dbh } \\
\quad(\mathrm{cm})\end{array}$ & $\begin{array}{c}\text { Ranking } \\
\text { for dbh } \dagger \ddagger\end{array}$ & $\begin{array}{l}\text { Mean dbh } \\
\quad(\mathrm{cm}) \S\end{array}$ & $\begin{array}{l}\text { Height } \\
\text { (m) }\end{array}$ & $\begin{array}{l}\text { Vigour } \\
(0-5)\end{array}$ & $\begin{array}{l}\text { Insect } \\
\text { resistance } \\
(0-5)\end{array}$ & $\begin{array}{l}\text { Stem } \\
\text { form } \\
(0-5)\end{array}$ & $\begin{array}{c}\text { Crown } \\
\text { form } \\
(0-5)\end{array}$ & $\begin{array}{c}\text { Branch } \\
\text { form } \\
(0-5)\end{array}$ & $\begin{array}{l}\text { Utility } \\
\text { rating } \\
(0-5)\end{array}$ & $\begin{array}{c}\text { Combined } \\
\text { subjective } \\
\text { ranking } \dagger\end{array}$ \\
\hline $\begin{aligned} \text { Acacia dealbata } & \text { longifolia }\end{aligned}$ & 2 & 29.4 & $7(3)$ & 26.2 & 10.4 & 4.4 & 3.9 & 2.7 & 2.2 & 2.3 & 2.8 & 32 \\
\hline var. sophorae & 1 & 12.0 & 46 & 10.0 & 3.9 & 4.0 & 3.8 & 0.6 & 1.0 & 3.0 & 1.8 & 47 \\
\hline mearnsii & 2 & 25.4 & 17 & 19.8 & 8.5 & 2.4 & 0.5 & 2.2 & 1.9 & 1.9 & 0.8 & 55 \\
\hline melanoxylon & 1 & 20.6 & $31=$ & 18.0 & 7.0 & 4.4 & 4.0 & 3.0 & 2.4 & 2.4 & 3.0 & 28 \\
\hline parramattensis & 1 & 19.2 & 35 & 17.1 & 10.6 & 4.0 & 4.0 & 2.0 & 1.0 & 1.5 & 1.6 & 48 \\
\hline \multicolumn{13}{|l|}{ Casuarina } \\
\hline cunninghamiana & 1 & 8.0 & 54 & $6.6^{+}$ & 5.8 & 2.0 & 5.0 & 3.4 & 3.4 & 3.6 & 1.0 & $29=$ \\
\hline glauca & 1 & 9.8 & 51 & 7.4 & 6.4 & 2.0 & 4.8 & 3.6 & 4.2 & 3.8 & 1.0 & 27 \\
\hline littoralis & 1 & 8.6 & - & 8.6 & 5.7 & 2.7 & 5.0 & 3.0 & 3.0 & 3.0 & 1.3 & - \\
\hline stricta & 1 & 14.4 & 43 & 11.6 & 5.7 & 4.0 & 5.0 & 2.0 & 2.0 & 3.0 & 1.8 & 33 \\
\hline torulosa & 1 & 7.3 & - & 7.3 & - & 2.3 & 5.0 & 3.3 & 3.3 & 3.3 & 1.3 & - \\
\hline Eucalyptus agglomerata & 1 & 26.2 & 15 & 21.0 & 7.9 & 4.4 & 4.0 & 3.4 & 3.6 & 3.0 & 3.0 & $15=$ \\
\hline aggregata & 1 & 17.8 & 40 & 16.8 & 7.2 & 2.8 & 3.3 & 3.3 & 3.0 & 2.8 & 1.8 & 36 \\
\hline amplifolia & 1 & 10.2 & 49 & 8.8 & 5.1 & 3.4 & 3.0 & 3.6 & 3.2 & 3.0 & 1.0 & 35 \\
\hline amygdalina & 1 & 27.7 & $13(5=)$ & 25.1 & 10.5 & 4.8 & 4.6 & 3.4 & 4.2 & 2.8 & 4.0 & 2 \\
\hline badjensis & 1 & 19.0 & $36=$ & 16.6 & 5.2 & 4.5 & 2.6 & 2.2 & 1.5 & 2.2 & 1.6 & 44 \\
\hline barberi & 1 & 9.6 & 52 & 8.8 & 4.0 & 1.4 & 1.8 & 2.0 & 3.0 & 3.2 & 0.2 & 52 \\
\hline blaxlandii & 1 & 14.0 & 45 & 12.0 & 5.1 & 2.6 & 3.4 & 2.8 & 2.8 & 3.2 & 1.0 & $41=$ \\
\hline botryoides & 1 & 27.8 & $12(2)$ & 26.8 & 11.7 & 4.8 & 3.6 & 3.6 & 2.8 & 2.4 & 3.2 & 23 \\
\hline bot. $\times$ saligna & 1 & 27.0 & $14(5=)$ & 25.1 & 12.4 & 5.0 & 4.0 & 4.0 & 3.2 & 2.8 & 3.4 & $10=$ \\
\hline brookeriana & 2 & 22.2 & $24(36)$ & $17.0^{+}$ & 9.5 & 4.1 & 2.5 & 3.9 & 4.0 & 3.5 & 2.7 & $20=$ \\
\hline camaldulensis & 2 & 6.7 & 55 & 6.6 & 4.4 & 1.8 & 1.7 & 3.4 & 3.7 & 3.0 & 0.7 & $45=$ \\
\hline camphora & 2 & 15.4 & 41 & 13.4 & 5.2 & 2.3 & 2.5 & 2.6 & 2.9 & 2.7 & 0.9 & 49 \\
\hline cinerea & 2 & 20.5 & $33(21=)$ & 19.6 & 5.0 & 3.0 & 3.4 & 3.0 & 2.6 & 3.0 & 1.1 & 39 \\
\hline cladocalyx & 2 & 29.2 & 8 & 24.8 & 13.4 & 4.8 & 4.0 & 3.8 & 4.0 & 3.0 & 3.5 & 6 \\
\hline coccifera & 2 & 14.2 & 44 & 13.1 & 5.3 & 3.2 & 3.4 & 2.4 & 3.2 & 2.8 & 1.0 & 40 \\
\hline cordata & 1 & 22.6 & $21=(30 \Rightarrow)$ & 18.0 & 10.1 & 5.0 & 4.2 & 3.8 & 3.2 & 3.2 & 3.6 & 7 \\
\hline cypellocarpa & 3 & 18.4 & $39(32=)$ & 17.6 & 6.6 & 2.8 & 1.7 & 3.0 & 2.9 & 2.5 & 0.7 & 50 \\
\hline deanei & 1 & 21.8 & $25(32=)$ & 17.6 & 9.4 & 4.4 & 3.6 & 3.4 & 3.0 & 2.7 & 2.6 & 25 \\
\hline dalrympleana & 6 & 12.2 & - & 12.2 & 4.2 & 1.6 & 2.0 & 2.7 & 3.1 & 3.1 & 0.9 & - \\
\hline delegatensis & 7 & 30.2 & $5=(13)$ & $22.0^{+++}$ & 12.4 & 4.7 & 4.1 & 4.1 & 3.3 & 3.2 & 3.9 & 5 \\
\hline dunnii & 3 & 15.5 & - & 13.2 & 6.6 & 2.5 & 2.5 & 3.0 & 3.0 & 3.0 & 0.0 & - \\
\hline elata & 2 & 22.4 & 23 & 19.0 & 8.5 & 4.7 & 3.7 & 3.2 & 2.9 & 2.8 & 2.5 & 25 \\
\hline fastigata & 4 & 34.6 & 1 & $29.2^{++}$ & 11.4 & 5.0 & 4.0 & 4.2 & 2.4 & 2.4 & 3.4 & $15=$ \\
\hline fraxinoides & 3 & 30.2 & $5=$ & $25.8^{+}$ & 13.1 & 4.8 & 4.0 & 3.6 & 3.4 & 2.9 & 3.3 & 12 \\
\hline globoidea & 1 & 28.2 & 11 & 24.4 & 8.6 & 4.9 & 4.0 & 3.0 & 3.0 & 3.3 & 3.0 & 19 \\
\hline johnstonii & 2 & 11.2 & 47 & 10.6 & 4.2 & 2.6 & 1.9 & 3.4 & 3.8 & 3.5 & 0.6 & $41=$ \\
\hline
\end{tabular}


TABLE 1 (Cont'd)

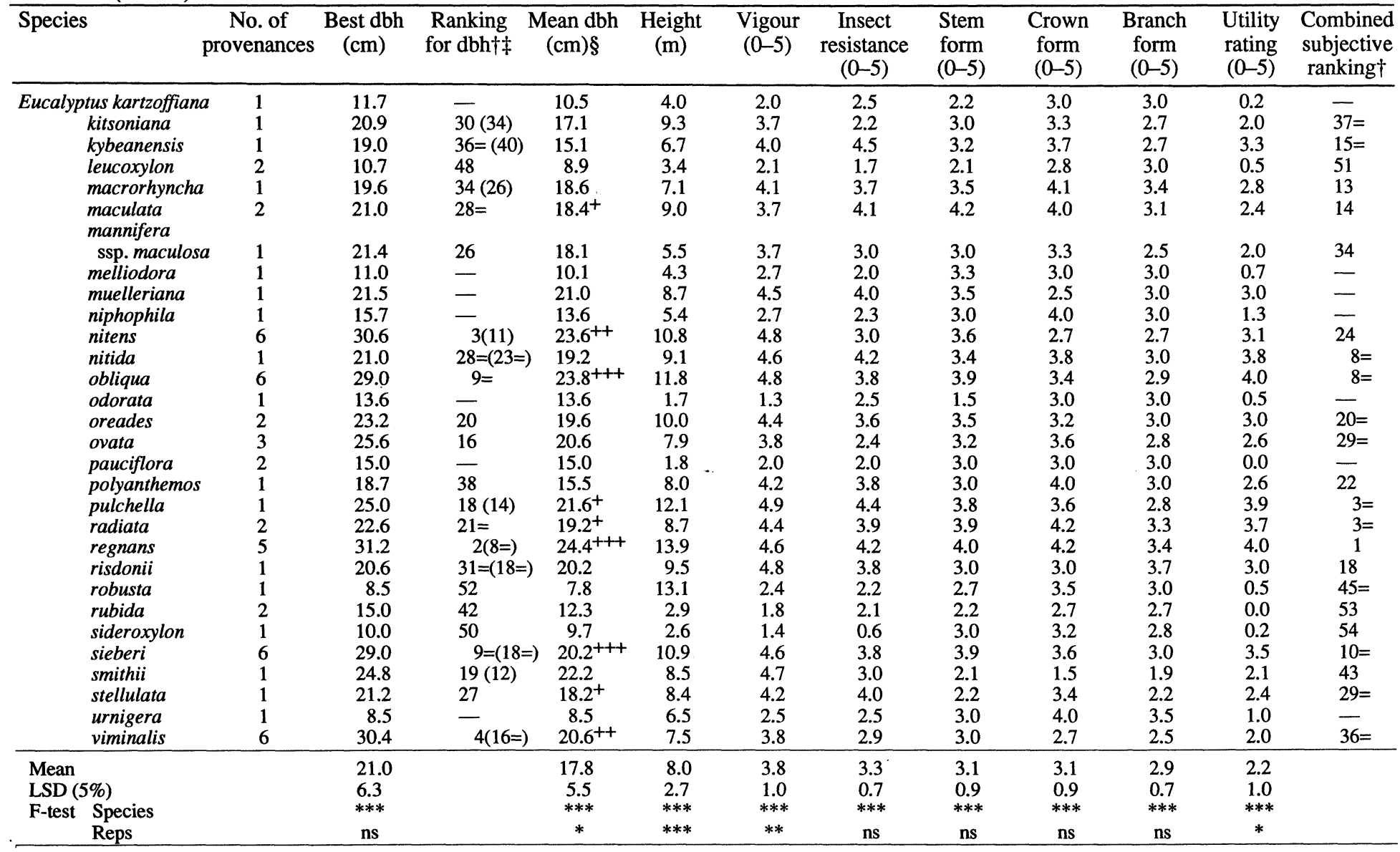

Notes: $\quad$ The rankings in parentheses are for mean dbh where that differs from best tree dbh ranking by more than one standard deviation (3.5) of all the differences. Unranked species were present in fewer than four replications and were not included in analyses of variance.

$\&$ Means were based on 5-15 trees, except those marked + which were based on 15-24 trees, ++ on 25-34, and +++ on 35-44 
TABLE 2-Assessment of Eucalyptus, Acacia, and Casuarina species in the Kahuiti trial, 12 years after planting

\begin{tabular}{|c|c|c|c|c|c|c|c|c|c|c|c|c|c|}
\hline Species & & $\begin{array}{l}\text { No. of } \\
\text { rovenances }\end{array}$ & $\begin{array}{l}\text { Mean dbh } \\
(\mathrm{cm}) \dagger\end{array}$ & $\begin{array}{l}\text { Ranking } \\
\text { for dbh } \ddagger\end{array}$ & $\begin{array}{l}\text { Height } \\
\text { (m) }\end{array}$ & $\begin{array}{l}\text { Survival } \\
(\%) \S\end{array}$ & $\begin{array}{l}\text { Vigour } \\
(0-5)\end{array}$ & $\begin{array}{c}\text { Insect } \\
\text { resistance } \\
(0-5)\end{array}$ & $\begin{array}{l}\text { Stem } \\
\text { form } \\
(0-5)\end{array}$ & $\begin{array}{c}\text { Crown } \\
\text { form } \\
(0-5)\end{array}$ & $\begin{array}{c}\text { Branch } \\
\text { form } \\
(0-5)\end{array}$ & $\begin{array}{l}\text { Utility } \\
\text { rating } \\
(0-5)\end{array}$ & $\begin{array}{c}\text { Combined } \\
\text { subjective } \\
\text { ranking } \ddagger\end{array}$ \\
\hline \multirow[t]{5}{*}{ Acacia } & $\begin{array}{l}\text { dealbata } \\
\text { longifolia }\end{array}$ & 2 & $14.3^{++}$ & 16 & 6.4 & 57 & 2.7 & 4.0 & 2.1 & 1.8 & 1.8 & 1.6 & 26 \\
\hline & var. sophorae & 1 & $13.7^{+}$ & 18 & 3.4 & $66(55)$ & 4.0 & 3.4 & 0.2 & 0.3 & 0.2 & 1.0 & 46 \\
\hline & mearnsii & 1 & $24.6^{+}$ & 1 & 8.1 & 60 & 3.2 & 2.1 & 0.7 & 0.0 & 0.0 & 0.6 & 48 \\
\hline & melanoxylon & 1 & $15.4^{+++}$ & 8 & 7.3 & 93 & 4.2 & 4.0 & 2.8 & 2.4 & 1.6 & 2.4 & 12 \\
\hline & parramattensis & 1 & 16.9 & $5=$ & 6.9 & $59(50)$ & 3.4 & 3.8 & 1.2 & 0.6 & 0.3 & 1.0 & $43=$ \\
\hline \multicolumn{14}{|c|}{ Casuarina } \\
\hline & cunninghamiana & 1 & 9.0 & 39 & 6.0 & $28(23)$ & 2.5 & 5.0 & 3.5 & 2.8 & 3.1 & 1.2 & 8 \\
\hline & glauca & 1 & 3.2 & - & 2.2 & $21(13)$ & 1.3 & 4.3 & 2.3 & 2.3 & 2.3 & 0.0 & - \\
\hline \multicolumn{2}{|c|}{ Eucalyptus agglomerata } & 1 & $9.5^{+}$ & $36=$ & 5.9 & $50(43)$ & 2.8 & 3.5 & 3.2 & 3.8 & 3.4 & 1.0 & 11 \\
\hline & aggregata & 1 & $11.9^{+}$ & 27 & 8.6 & 42 & 2.6 & 2.8 & 2.4 & 3.2 & 3.0 & 0.8 & $22=$ \\
\hline & amplifolia & 1 & $5.5^{+}$ & $47=$ & 3.8 & 70 & 1.7 & 2.0 & 2.7 & 3.3 & 3.2 & 0.4 & 28 \\
\hline & amygdalina & 1 & $14.8^{+}$ & $10=$ & 9.5 & $56(47)$ & 3.0 & 3.3 & 3.5 & 4.0 & 1.9 & 3.2 & 5 \\
\hline & badjensis & 1 & $9.9^{++}$ & $33=$ & 4.7 & 90 & 2.5 & 1.5 & 2.7 & 2.2 & 2.0 & 0.4 & 39 \\
\hline & barberi & 1 & $8.9^{+}$ & 40 & 4.9 & 55 & 1.8 & 1.4 & 1.6 & 2.0 & 2.2 & 0.0 & 47 \\
\hline & botryoides & 1 & $19.4^{+}$ & $2=$ & 8.6 & $90(80)$ & 3.8 & 3.0 & 3.0 & 2.4 & 1.6 & 2.0 & $16=$ \\
\hline & bot. $\times$ saligna & 1 & $14.8^{++}$ & $10=$ & 8.2 & 80 & 3.0 & 3.0 & 3.2 & 2.6 & 2.0 & 2.2 & 15 \\
\hline & brookeriana & 2 & $16.9^{++}$ & $5=$ & 9.3 & $85(81)$ & 3.2 & 1.9 & 3.7 & 3.5 & 2.9 & 1.9 & 13 \\
\hline & camaldulensis & 2 & $7.2^{+}$ & $43=$ & 3.9 & $71(40)$ & 1.7 & 2.0 & 2.5 & 2.8 & 2.9 & 0.1 & 34 \\
\hline & camphora & 2 & $12.2^{++}$ & $25=$ & 7.0 & $80(70)$ & 2.7 & 2.2 & 1.9 & 2.4 & 2.2 & 0.4 & 35 \\
\hline & cinerea & 2 & $15.3^{+}$ & 9 & 5.3 & $70(65)$ & 2.5 & 2.6 & 2.3 & 1.9 & 1.9 & 0.4 & $36=$ \\
\hline & cladocalyx & 2 & 12.2 & - & 8.3 & $50(15)$ & 2.3 & 3.7 & 4.0 & 3.3 & 3.0 & 1.3 & - \\
\hline & coccifera & 2 & 8.1 & 41 & 3.9 & $57(23)$ & 2.0 & 2.8 & 2.5 & 3.0 & 2.7 & 0.5 & 27 \\
\hline & cordata & 1 & $17.9^{++}$ & 4 & 11.4 & 63 & 4.0 & 3.0 & 3.8 & 3.6 & 3.2 & 3.2 & 2 \\
\hline & cypellocarpa & 3 & $14.5^{++}$ & 15 & 5.7 & $63(50)$ & 2.3 & 1.6 & 2.2 & 2.0 & 1.8 & 0.4 & $43=$ \\
\hline & dalrympleana & 6 & $13.5^{++++}$ & 20 & 5.5 & $86(79)$ & 2.2 & 1.8 & 2.6 & 3.3 & 2.2 & 0.8 & 30 \\
\hline & deanei & 1 & $12.3^{++}$ & 24 & 6.4 & 70 & 2.4 & 2.4 & 2.8 & 2.4 & 2.2 & 0.6 & 31 \\
\hline & delegatensis & 7 & $12.9^{++}$ & 23 & 8.5 & $58(14)$ & 2.9 & 3.2 & 3.7 & 3.8 & 3.4 & 2.5 & 3 \\
\hline & dunnii & 3 & $6.6^{+}$ & 45 & 3.5 & $65(36)$ & 1.7 & 1.8 & 2.4 & 2.5 & 2.7 & 0.0 & 40 \\
\hline & elata & 2 & $9.9^{+}$ & $33=$ & 5.1 & $58(35)$ & 2.4 & 3.2 & 3.0 & 3.2 & 2.8 & 1.2 & $16=$ \\
\hline & fastigata & 4 & $14.7^{++++}$ & 14 & 9.0 & $67(59)$ & 3.1 & 3.2 & 3.7 & 3.5 & 3.2 & 1.9 & 6 \\
\hline & fraxinoides & 3 & $14.8^{++}$ & $10=$ & 7.7 & $63(45)$ & 3.1 & 3.2 & 3.4 & 3.7 & 2.6 & 1.9 & 10 \\
\hline & globoidea & 1 & $12.2^{+}$ & $25=$ & 4.7 & $50(43)$ & 3.1 & 3.5 & 2.2 & 3.0 & 1.9 & 1.3 & $20=$ \\
\hline & johnstonii & 2 & $9.7^{++}$ & 35 & 4.6 & $80(73)$ & 2.2 & 1.2 & 3.5 & 4.2 & 3.9 & 0.8 & $16=$ \\
\hline
\end{tabular}


TABLE 2 (Cont'd)

\begin{tabular}{|c|c|c|c|c|c|c|c|c|c|c|c|c|}
\hline \multicolumn{2}{|c|}{$\begin{array}{lc}\text { Species } & \begin{array}{c}\text { No. of } \\
\text { provenances }\end{array} \\
\end{array}$} & \multirow{2}{*}{$\frac{\begin{array}{c}\text { Mean dbh } \\
(\mathrm{cm}) \dagger\end{array}}{14.3}$} & \multirow{2}{*}{$\begin{array}{l}\text { Ranking } \\
\text { for dbh } \ddagger \\
-\end{array}$} & \multirow{2}{*}{$\begin{array}{l}\begin{array}{c}\text { Height } \\
(\mathrm{m})\end{array} \\
7.2\end{array}$} & \multirow{2}{*}{$\begin{array}{c}\begin{array}{c}\text { Survival } \\
(\%) \S\end{array} \\
88(53)\end{array}$} & \multirow{2}{*}{$\begin{array}{c}\begin{array}{l}\text { Vigour } \\
(0-5)\end{array} \\
2.7\end{array}$} & \multirow{2}{*}{$\begin{array}{c}\begin{array}{c}\text { Insect } \\
\text { resistance } \\
(0-5)\end{array} \\
2.0\end{array}$} & \multirow{2}{*}{$\begin{array}{c}\begin{array}{l}\text { Stem } \\
\text { form } \\
(0-5)\end{array} \\
2.0\end{array}$} & \multirow{2}{*}{$\begin{array}{c}\begin{array}{c}\text { Crown } \\
\text { form } \\
(0-5)\end{array} \\
2.3\end{array}$} & \multirow{2}{*}{$\begin{array}{c}\begin{array}{c}\text { Branch } \\
\text { form } \\
(0-5)\end{array} \\
2.0\end{array}$} & \multirow{2}{*}{$\begin{array}{l}\text { Utility } \\
\text { rating } \\
(0-5)\end{array}$} & \multirow{2}{*}{$\begin{array}{c}\text { Combined } \\
\text { subjective } \\
\text { ranking } \ddagger\end{array}$} \\
\hline \multirow{4}{*}{$\begin{array}{c}\text { Eucalyptus kartzoffiana } \\
\text { kitsoniana } \\
\text { leucoxylon } \\
\text { macrorhyncha } \\
\text { mannifera }\end{array}$} & 1 & & & & & & & & & & & \\
\hline & 1 & $13.6^{+++}$ & 19 & 8.8 & 95 & 3.0 & 2.4 & 2.6 & 2.2 & 2.8 & 1.2 & 25 \\
\hline & 2 & $6.3^{++}$ & 46 & 3.6 & $56(48)$ & 2.0 & 2.5 & 2.0 & 2.3 & 2.6 & 0.2 & $36=$ \\
\hline & 1 & 10.6 & 32 & 6.2 & 37 & 2.2 & 3.0 & 2.8 & 3.6 & 2.6 & 0.6 & $22=$ \\
\hline ssp. maculosa & 1 & $8.1^{+}$ & - & 5.0 & $54(32)$ & 1.7 & 1.7 & 2.0 & 2.7 & 2.3 & 0.3 & - \\
\hline melliodora & 1 & 9.5 & $36=$ & 4.2 & 28 & 2.0 & 2.6 & 1.8 & 2.8 & 2.6 & 0.4 & 33 \\
\hline muelleriana & 1 & 19.8 & - & 8.0 & $63(25)$ & 4.0 & 3.0 & 2.5 & 3.0 & 2.0 & 1.5 & - \\
\hline niphophila & 1 & $11.2^{+++}$ & 29 & 5.5 & 68 & 2.6 & 3.0 & 2.6 & 4.0 & 2.6 & 1.0 & $16=$ \\
\hline nitens & 6 & $14.8^{++++}$ & $10=$ & 9.2 & $75(60)$ & 3.2 & 2.5 & 3.5 & 2.6 & 2.7 & 2.2 & 14 \\
\hline nitida & 1 & 18.2 & - & 9.0 & $75(30)$ & 3.0 & 3.0 & 2.5 & 2.5 & 2.5 & 1.5 & - \\
\hline obliqua & 6 & $19.4^{++++}$ & $2=$ & 11.0 & $63(43)$ & 2.9 & 2.9 & 3.9 & 3.7 & 3.0 & 2.6 & 4 \\
\hline oreades & 2 & 11.3 & - & 5.5 & $50(15)$ & 3.3 & 3.0 & 3.3 & 3.3 & 2.7 & 2.0 & - \\
\hline ovata & 3 & $10.9^{+}$ & 30 & 6.0 & $76(55)$ & 2.5 & 1.8 & 3.2 & 3.2 & 3.3 & 1.1 & $20=$ \\
\hline polyanthemos & 1 & $5.5^{+}$ & $47=$ & 4.3 & 45 & 2.2 & 2.8 & 2.2 & 2.6 & 2.8 & 0.2 & 29 \\
\hline pulchella & 1 & 18.4 & - & 11.3 & $71(43)$ & 3.7 & 4.0 & 3.3 & 3.3 & 2.0 & 3.0 & - \\
\hline radiata & 2 & $10.7^{+}$ & 31 & 5.4 & $57(55)$ & 2.6 & 3.0 & 3.3 & 3.8 & 3.4 & 1.9 & 9 \\
\hline regnans & 5 & $16.4^{++++}$ & 7 & 11.1 & $68(66)$ & 3.4 & 3.3 & 4.0 & 3.8 & 3.5 & 3.4 & 1 \\
\hline risdonii & 1 & $9.1^{++}$ & 38 & 5.8 & 80 & 1.8 & 3.2 & 1.8 & 2.0 & 2.4 & 0.2 & 38 \\
\hline robusta & 1 & $7.2^{+}$ & $43=$ & 3.2 & $78(65)$ & 1.5 & 1.3 & 2.2 & 2.8 & 2.2 & 0.2 & 42 \\
\hline rubida & 2 & $11.4^{++}$ & 28 & 5.7 & $75(63)$ & 2.1 & 2.4 & 1.7 & 1.9 & 2.1 & 0.1 & $43=$ \\
\hline sideroxylon & 1 & 8.6 & - & 3.2 & $46(28)$ & 1.7 & 2.3 & 1.7 & 2.7 & 2.7 & 0.0 & - \\
\hline sieberi & 6 & $14.1^{++}$ & 17 & 7.2 & $51(22)$ & 3.1 & 3.5 & 3.2 & 3.7 & 2.9 & 2.1 & 7 \\
\hline smithii & 1 & $7.7^{++}$ & 42 & 5.2 & 83 & 1.8 & 1.5 & 2.2 & 2.7 & 2.3 & 0.5 & 41 \\
\hline stellulata & 1 & $13.1^{++}$ & 22 & 6.7 & 69 & 3.0 & 2.9 & 2.3 & 3.1 & 2.3 & 1.2 & $22=$ \\
\hline viminalis & 6 & $13.4^{++++}$ & 21 & 6.5 & $76(62)$ & 2.6 & 1.9 & 2.6 & 2.6 & 2.2 & 0.8 & 32 \\
\hline Mean & & 12.3 & & 6.4 & $66(58)$ & 2.6 & 2.7 & 2.7 & 2.8 & 2.4 & 1.2 & \\
\hline LSD (5\%) & & 6.0 & & 2.5 & $27(26)$ & 0.9 & 0.8 & 0.8 & 0.8 & 0.9 & 1.0 & \\
\hline F-test Species & & $* * *$ & & $* * *$ & $* * *$ & $* * *$ & $* * *$ & $* * *$ & $* * *$ & $* * *$ & $* * *$ & \\
\hline Reps & & ns & & ns & $* * *$ & ns & ns & $*$ & ns & $*$ & ns & \\
\hline
\end{tabular}

Notes: $\dagger$ Means were based on a minimum of four trees, and those marked + on $15-24$ trees, ++ on $25-34,+++$ on $35-44$, and ++++ on more than 45 .

$\$$ Unranked species were present in fewer than four replications and were not included in analyses of variance. 
TABLE 3-Comparison of 1984 and 1991 means

\begin{tabular}{lcccccccc}
\hline Site & Year & $\begin{array}{c}\text { Dbh } \\
(\mathrm{cm})\end{array}$ & $\begin{array}{c}\text { Height } \\
(\mathrm{m})\end{array}$ & $\begin{array}{c}\text { Actual } \\
\text { survival } \\
(\%)\end{array}$ & $\begin{array}{c}\text { Insect } \\
\text { resistance } \\
(0-5)\end{array}$ & $\begin{array}{c}\text { Stem } \\
\text { form } \\
(0-5)\end{array}$ & $\begin{array}{c}\text { Crown } \\
\text { form } \\
(0-5)\end{array}$ & $\begin{array}{c}\text { Branch } \\
\text { form } \\
(0-5)\end{array}$ \\
\hline Pakaraka & 1984 & 4.0 & 3.3 & 81 & $3.4 / 3.8^{*}$ & 2.4 & 1.8 & 2.0 \\
& 1991 & 17.8 & 8.0 & NA & 3.3 & 3.1 & 3.1 & 2.9 \\
\multirow{3}{*}{ Kahuiti } & 1984 & 3.1 & 2.9 & $66 \dagger$ & $4.0 / 4.6^{*}$ & 2.4 & 1.9 & 2.1 \\
& 1991 & 12.3 & 6.4 & $63 \dagger$ & 2.7 & 2.7 & 2.8 & 2.4 \\
\hline
\end{tabular}

* Insect resistance was rated separately for Paropsis charybdis and leafroller caterpillar.

$\dagger$ The fifth replication at Kahuiti was not included in these survival percentages.

not a eucalypt. The upper grouping at Kahuiti comprised 13 species, again one of which (A. melanoxylon $\mathrm{R}$. Br.) is not a eucalypt. Ten species were common to the upper groupings at both sites. Hathaway \& King (1986) recognised 15 top species at each site. Most of these remained in the upper groupings of 1991. Exceptions at Pakaraka were E. stellulata Sieb. ex DC. which was adjudged marginally below the upper grouping in 1991, and E. muelleriana Howitt which survived in only two replications. At Kahuiti exceptions were $E$. viminalis, $E$. deanei Maid., and E. camphora R.T. Baker, all assigned to the middle grouping in 1991, and unranked $E$. pulchella and E. kartzoffiana L. Johnson \& D. Blaxell*, both represented in only three replications in 1991.

Performance grouping summary data for the two sites on a plot basis are presented in Table 5. Within groupings the superiority of the Pakaraka site was evident for means of dbh, height, vigour, and insect resistance, and to a lesser extent for the growth habit ratings. However, for all factors within groupings there was a considerable range of variation, as evidenced by the size of the standard deviations and the range of the plot mean observations. "About average" was rated at a score of 3 . Of note is that mean vigour for the upper grouping at Pakaraka was close to the maximum rating score of 5, that insect resistance was apparently not a factor in differentiating between upper and middle groupings at Pakaraka, and that even the upper grouping at Kahuiti averaged less than 3.0 for the utility rating, i.e., was perceived to be of less than average usefulness for soil conservation plantings on such a hillslope.

Growth of E. regnans, the species which conformed best to the selection criteria over Pakaraka and Kahuiti combined, may be compared with a tended stand of the same species on a Rotorua site of one-sixth of a hectare, with freely draining sandy loam soil derived from pumice, and receiving $1440 \mathrm{~mm}$ annual rainfall. At 13 years when the stand density was the equivalent of $237 \mathrm{stems} / \mathrm{ha}$, mean height was $28.5 \mathrm{~m}$ and dbh was $40.7 \mathrm{~cm}$ (Wilcox \& Thulin 1979), twice the height and 1.3 times the mean best dbh at Pakaraka at 12 years, and well over double the corresponding means at Kahuiti (unthinned but of comparable density). The differences may be attributed to annual rainfall, incidence of drought, soil texture, and soil fertility.

Fourteen of the species and a hybrid of one in the Wairarapa trials were also represented (many of them by the same seedlots) in a potential production forestry eucalypt selection trial

* The accession attributed to E. kartzoffiana may be a variant of E. viminalis. 
TABLE 4-Performance groupings

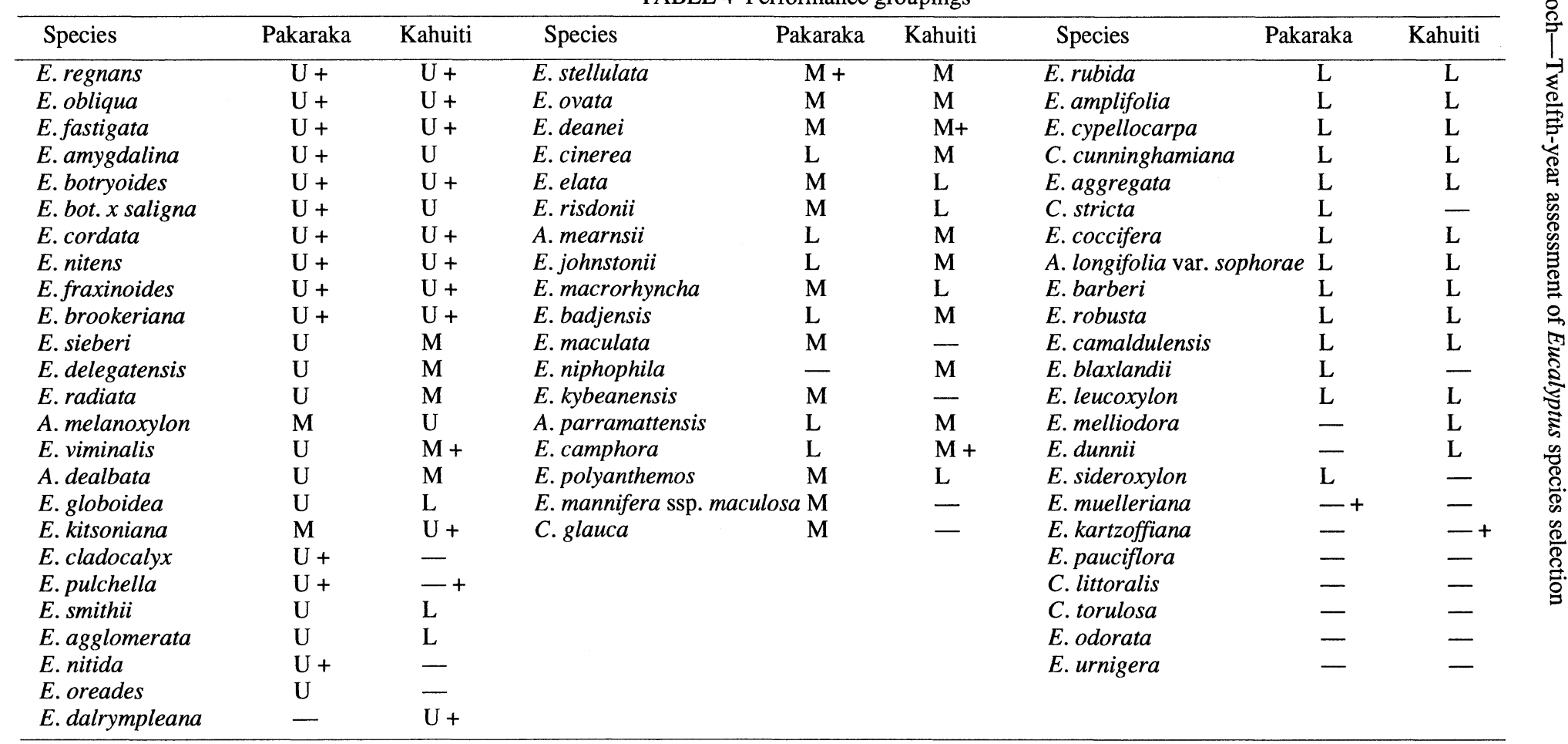

$\mathrm{U}=$ In upper grouping.

$\mathrm{M}=$ In middle grouping.

$\mathrm{L}=$ In lower grouping.

- = Unranked

$+\quad$ = In upper grouping of Hathaway \& King (1986). 
TABLE 5-Performance grouping summary data*

\begin{tabular}{|c|c|c|c|c|c|c|c|c|c|c|c|}
\hline Group/site & & $\begin{array}{l}\text { Best dbh } \\
\text { (cm) }\end{array}$ & $\begin{array}{l}\text { Mean dbh } \\
\quad(\mathrm{cm})\end{array}$ & $\begin{array}{l}\text { Height } \\
\text { (m) }\end{array}$ & $\begin{array}{c}\text { Survival } \\
(\%)\end{array}$ & $\begin{array}{l}\text { Vigour } \\
(0-5)\end{array}$ & $\begin{array}{l}\text { Insect } \\
\text { resistance } \\
(0-5)\end{array}$ & $\begin{array}{l}\text { Stem form } \\
\quad(0-5)\end{array}$ & $\begin{array}{l}\text { Crown form } \\
\quad(0-5)\end{array}$ & $\begin{array}{l}\text { Branch form } \\
\quad(0-5)\end{array}$ & $\begin{array}{l}\text { Utility rating } \\
\quad(0-5)\end{array}$ \\
\hline \multirow{2}{*}{ Upper } & Pak & $\begin{array}{c}27.4 \pm 5.5 \\
(15.0-41.0)\end{array}$ & $\begin{array}{c}22.6 \pm 5.0 \\
(14.0-36.0)\end{array}$ & $\begin{array}{c}10.7 \pm 2.6 \\
(4.5-18.4)\end{array}$ & NA & $\begin{array}{l}4.7 \pm 0.5 \\
(3.1-5)\end{array}$ & $\begin{array}{c}3.8 \pm 0.6 \\
(2-5)\end{array}$ & $\begin{array}{l}3.6 \pm 0.7 \\
(1.5-5)\end{array}$ & $\begin{array}{c}3.3 \pm 0.9 \\
(1-5)\end{array}$ & $\begin{array}{c}2.9 \pm 0.6 \\
(1.5-4.5)\end{array}$ & $\begin{array}{l}3.3 \pm 0.8 \\
(0.7-5)\end{array}$ \\
\hline & Kah & NA & $\begin{array}{c}15.9 \pm 4.9 \\
(6.3-29.7)\end{array}$ & $\begin{array}{c}9.0 \pm 2.6 \\
(3.2-14.0)\end{array}$ & $\begin{array}{c}76 \pm 23 \\
(20-100)\end{array}$ & $\begin{array}{c}3.2 \pm 0.8 \\
(1-5)\end{array}$ & $\begin{array}{c}2.9 \pm 0.7 \\
(1-4)\end{array}$ & $\begin{array}{l}3.4 \pm 0.7 \\
(0-4.2)\end{array}$ & $\begin{array}{c}3.1 \pm 0.8 \\
(0-4)\end{array}$ & $\begin{array}{c}2.6 \pm 0.8 \\
(1-4)\end{array}$ & $\begin{array}{c}2.2 \pm 1.1 \\
(0-5)\end{array}$ \\
\hline \multirow{2}{*}{ Middle } & Pak & $\begin{array}{c}20.2 \pm 5.5 \\
(6.0-35.0)\end{array}$ & $\begin{array}{c}17.1 \pm 4.9 \\
(4.0-24.0)\end{array}$ & $\begin{array}{c}7.9 \pm 2.4 \\
(1.5-13.5)\end{array}$ & NA & $\begin{array}{c}3.9 \pm 0.9 \\
(1-5)\end{array}$ & $\begin{array}{c}3.8 \pm 0.9 \\
(2-5)\end{array}$ & $\begin{array}{c}3.2 \pm 0.7 \\
(2-5)\end{array}$ & $\begin{array}{c}3.5 \pm 0.8 \\
(1-5)\end{array}$ & $\begin{array}{c}2.9 \pm 0.6 \\
(2-4)\end{array}$ & $\begin{array}{c}2.5 \pm 1.0 \\
(0-5)\end{array}$ \\
\hline & Kah & NA & $\begin{array}{c}13.3 \pm 4.9 \\
(6.4-23.6)\end{array}$ & $\begin{array}{c}6.3 \pm 1.7 \\
(2.0-10.0)\end{array}$ & $\begin{array}{c}69 \pm 22 \\
(25-100)\end{array}$ & $\begin{array}{l}2.7 \pm 0.5 \\
(1.5-4)\end{array}$ & $\begin{array}{c}2.6 \pm 1.0 \\
(0-4)\end{array}$ & $\begin{array}{c}2.5 \pm 0.9 \\
(0-4)\end{array}$ & $\begin{array}{c}2.7 \pm 1.3 \\
(0-5)\end{array}$ & $\begin{array}{c}2.3 \pm 1.1 \\
(0-5)\end{array}$ & $\begin{array}{l}1.1 \pm 0.9 \\
(0-3.5)\end{array}$ \\
\hline \multirow{2}{*}{ Lower } & Pak & $\begin{array}{c}14.2 \pm 6.3 \\
(4.0-33.0)\end{array}$ & $\begin{array}{c}12.6 \pm 5.4 \\
(4.0-24.0)\end{array}$ & $\begin{array}{c}5.4 \pm 2.5 \\
(1.8-14.0)\end{array}$ & NA & $\begin{array}{c}2.7 \pm 1.2 \\
(1-5)\end{array}$ & $\begin{array}{c}2.7 \pm 1.4 \\
(0-5)\end{array}$ & $\begin{array}{c}2.6 \pm 1.0 \\
(0-5)\end{array}$ & $\begin{array}{c}2.7 \pm 1.0 \\
(0-5)\end{array}$ & $\begin{array}{c}2.8 \pm 0.7 \\
(0-5)\end{array}$ & $\begin{array}{c}0.9 \pm 0.9 \\
(0-3)\end{array}$ \\
\hline & Kah & NA & $\begin{array}{c}9.1 \pm 4.7 \\
(1.0-25.9)\end{array}$ & $\begin{array}{c}4.9 \pm 2.0 \\
(1.5-8.5)\end{array}$ & $\begin{array}{c}58 \pm 24 \\
(13-100)\end{array}$ & $\begin{array}{c}2.2 \pm 0.8 \\
(1-4)\end{array}$ & $\begin{array}{c}2.6 \pm 1.0 \\
(1-5)\end{array}$ & $\begin{array}{c}2.6 \pm 0.9 \\
(0-4)\end{array}$ & $\begin{array}{c}2.6 \pm 0.9 \\
(0-5)\end{array}$ & $\begin{array}{c}2.5 \pm 0.9 \\
(0-4)\end{array}$ & $\begin{array}{c}0.5 \pm 0.7 \\
(0-3)\end{array}$ \\
\hline
\end{tabular}

* Mean \pm one standard deviation / (minimum-maximum). 
of 20 species and two hybrids planted in 1978 on three central North Island pumiceland sites ranging from "warm" (70 m a.s.1.) to "cold" (920 m a.s.1.) (Johnson \& Wilcox 1989). After 9 years, best on the warm site was E. saligna (the nearest Wairarapa equivalent being the upper ranked $E$. botryoides $\times$ saligna) and best on the cold site was $E$. dendromorpha (Blakely) L. Johnson \& D. Blaxell (unrepresented in Wairarapa). The most adaptably performing species over the three pumiceland sites were E. regnans and E. fastigata, followed by $E$. delegatensis, $E$. fraxinoides, and $E$. obliqua; $E$. nitens apart from $P$. charybdis damage; and $E$. oreades on the warm site.

Thus for the species in common, upper rankings in both sets of trials were similar, and likewise for the "losers" (Johnson \& Wilcox 1989) at the other end of the scale, E. cypellocarpa L. Johnson, E. dunnii, E. gunnii Maid., and E. johnstonii Maid. Species attaining an upper grouping on the Wairarapa sites but failing on the pumiceland, and therefore apparently better adapted to the former, were $E$. sieberi, $E$. viminalis, and $E$. dalrympleana Maid. Over the 15 species, the trees on the pumiceland sites were on average $4 \mathrm{~m}$ taller despite the Wairarapa trees being 3 years older.

\section{Provenances}

Comparison of provenances within species indicated significant differences at Pakaraka for $E$. sieberi (dbh and height), E. delegatensis (height), E. obliqua (height), E. radiata (dbh and vigour), E. brookeriana (height, vigour, and crown form), E. viminalis (vigour), and $E$. nitens (utility rating). At Kahuiti there were significant differences for $E$. dalrympleana (vigour, crown form, and utility rating) and E. fastigata (utility rating). The differences are shown in Tables 6-14 with mean separation at the 5\% significance level. The general form of the differences was that the best and worst provenances differed significantly from each other but neither differed significantly from intermediate provenances.

The $E$. regnans provenances each represented one of five of the nine provenance groupings identified by Wilcox (1982a) in an assessment of 36 provenances 3 years after planting on two central North Island sites. Unfortunately, Wilcox's "best overall provenance" -Tokoroa, NZ, of Tasmanian origin, FRI77/2062-was not represented. Wilcox's "best all-round native provenance group" was "interior south Tasmania", typified by the Moogara provenance HO68/614. Although differences were not significant for individual parameters, the Moogara provenance was the top-ranking provenance across all parameters and both sites in the Wairarapa assessment. Ranking equally next were Rangiwahia (NZ, of Victorian origin)(FRI77/2063), Franklin(Tasmania)(HO74/815), and Ruapuna(Canterbury)(FRI77/ 2064), representing mid to lower placed provenance groupings in Wilcox's North Island assessment. However, in a trial of the same 36 provenances at Longwood Forest, Southland, assessed 6 years after planting (Wilcox et al. 1985), the Moogara provenance ranked worst of the four (33 out of 36) and the Franklin provenance the best (12 out of 36). The malformation Wilcox discerned for the Rangiwahia provenance, otherwise one of the fastest growing on the central North Island sites, was not evident on the Wairarapa sites.

The order of listing for $E$. nitens provenances in Table 12 represented the order of ranking across all parameters at Pakaraka, although only the utility rating differences were significant. This tended to confirm the superiority of central Victoria (Rubicon, Toorongo, and Macalister) provenances over eastern Victoria (Errinundra Plateau) and New South Wales provenances, as documented by King \& Wilcox (1988) in a progeny trial of 83 E. nitens 
TABLE 6-Eucalyptus sieberi provenance differences at Pakaraka

\begin{tabular}{clll}
\hline Accession & \multicolumn{1}{c}{ Provenance } & \multicolumn{2}{c}{ Dbh (cm) Height (cm) } \\
\hline 1197 & Pikes Saddle, N.S.W. 930 m a.s.l. CSIRO seedlot No. 12119 & $27.3 \mathrm{a}$ & $11.3 \mathrm{ab}$ \\
2436 & Powelltown, Victoria. NZ FRISI/8 HO/67/598 & $23.1 \mathrm{ab}$ & $12.5 \mathrm{a}$ \\
2435 & Nerrigundah, N.S.W. 305 m a.s.l. CSIRO seedlot No. 11971 & $22.6 \mathrm{ab}$ & $10.9 \mathrm{ab}$ \\
2433 & Fingal, Tasmania. 450 m a.s.l. CSIRO seedlot No. 9983 & $22.0 \mathrm{ab}$ & $11.5 \mathrm{ab}$ \\
2434 & Newnes, N.S.W. 1070 m a.s.l. CSIRO seedlot No. 10350 & $21.0 \mathrm{ab}$ & $11.3 \mathrm{ab}$ \\
2437 & Orbost, Victoria. 183 m a.s.l. CSIRO seedlot No. 12103 & $16.9 \mathrm{ab}$ & $10.4 \mathrm{ab}$ \\
1196 & Erica, Victoria. 520 m a.s.l. CSIRO seedlot No. 12391 & $15.2 \mathrm{~b}$ & $8.4 \mathrm{~b}$ \\
\hline
\end{tabular}

TABLE 7-Eucalyptus delegatensis provenance differences at Pakaraka

\begin{tabular}{clc}
\hline Accession & \multicolumn{1}{c}{ Provenance } & Height (m) \\
\hline 1975 & Swift Ck. near Omeo, Victoria. NZ FRI DE/167 HO/0/77/13 & $14.5 \mathrm{a}$ \\
1973 & Maggs Mt, Tasmania. 820 m a.s.1. NZ FRI DE/141 FRI78/2260 & $13.8 \mathrm{a}$ \\
1972 & Golden Downs Forest, Nelson. FRI DE/42 NN/0/75/03 & $13.0 \mathrm{ab}$ \\
1974 & Fingal Tier, Tasmania. 760 m a.s.1. NZ FRI DE/156 FRI78/2274 & $10.1 \mathrm{~b}$ \\
\hline
\end{tabular}

TABLE 8-Eucalyptus obliqua provenance differences at Pakaraka

\begin{tabular}{clc}
\hline Accession & \multicolumn{1}{c}{ Provenance } & Height $(\mathrm{m})$ \\
\hline 1185 & Tasmania seedlot 120 collected by C. Matheson & $14.0 \mathrm{a}$ \\
1337 & Mawbanna, Tasmania seedlot 140 collected by C. Matheson & $13.7 \mathrm{ab}$ \\
2456 & Otway Ranges, Victoria. NZ FRI OB/93 HO/0/78/19 & $12.7 \mathrm{ab}$ \\
2459 & Nietta, Tasmania. NZ FRI OB/23 FRI77/2120 & $12.2 \mathrm{ab}$ \\
2458 & Powelltown, Victoria. NZ FRI OB/25 FRI77/2122 & $10.2 \mathrm{~b}$ \\
\hline
\end{tabular}

TABLE 9-Eucalyptus radiata provenance differences at Pakaraka

\begin{tabular}{clcc}
\hline Accession & \multicolumn{1}{c}{ Provenance } & $\begin{array}{c}\text { Dbh } \\
(\mathrm{cm})\end{array}$ & $\begin{array}{c}\text { Vigour } \\
(0-5)\end{array}$ \\
\hline 1257 & Daylesford District, Kangaroo Ck, Victoria. 716 m a.s.l. & $22.6 \mathrm{a}$ & $4.8 \mathrm{a}$ \\
1190 & East Queanbeyan, N.S.W. $900 \mathrm{~m}$ a.s.l. CSIRO seedlot No. 12169 & $15.8 \mathrm{~b}$ & $4.2 \mathrm{~b}$ \\
\hline
\end{tabular}

TABLE 10-Eucalyptus brookeriana provenance differences at Pakaraka

\begin{tabular}{clccc}
\hline Accession & \multicolumn{1}{c}{ Provenance } & $\begin{array}{l}\text { Dbh } \\
(\mathrm{cm})\end{array}$ & $\begin{array}{c}\text { Vigour } \\
(0-5)\end{array}$ & $\begin{array}{c}\text { Crown form } \\
(0-5)\end{array}$ \\
\hline 1112 & $\begin{array}{l}\text { Road to Sea Elephant, King Is., } \\
\text { Tasmania. CSIRO seedlot No. B5866 }\end{array}$ & 11.3 & $4.8 \mathrm{a}$ & $3.4 \mathrm{~b}$ \\
& $\begin{array}{l}\text { Fingal, Tasmania. 520 m a.s.l. } \\
\text { CSIRO seedlot No. 9985 }\end{array}$ & $6.9 \mathrm{~b}$ & $3.4 \mathrm{~b}$ & $4.6 \mathrm{a}$ \\
\hline
\end{tabular}


TABLE 11-Eucalyptus viminalis provenance differences at Pakaraka

\begin{tabular}{clc}
\hline Accession & \multicolumn{1}{c}{ Provenance } & Vigour (0-5) \\
\hline 2089 & Bruthen, Victoria. 900 m a.s.l. CSIRO seedlot No. S11743 & $5.0 \mathrm{a}$ \\
2400 & Cygnet, Tasmania. 30 m a.s.l. & $3.9 \mathrm{~b}$ \\
2246 & Rotorua. NZ FRI VI/1 FRI76/2053 & $3.1 \mathrm{~b}$ \\
2248 & Kingston, Tasmania. NZ FRI VI/3 HO/0/76/07 & $3.0 \mathrm{~b}$ \\
\hline
\end{tabular}

TABLE 12-Eucalyptus nitens provenance differences at Pakaraka

\begin{tabular}{cll}
\hline Accession & \multicolumn{1}{c}{ Provenance } & $\begin{array}{c}\text { Utility rating } \\
(0-5)\end{array}$ \\
\hline 2451 & Rubicon provenance, Blue Ra., Victoria. NZ FRI NI/124 HO/0/78/17 & $3.9 \mathrm{a}$ \\
2452 & Toorongo provenance, Victoria. NZ FRI NI/126 FRI78/2153 & $3.0 \mathrm{ab}$ \\
2453 & Macalister provenance, Victoria. NZ FRI NI/127 FRI78/2154 & $3.0 \mathrm{ab}$ \\
2454 & Errinundra Plateau, Bendoc, Victoria. NZ FRI NI/60 FRI76/2009 & $3.0 \mathrm{ab}$ \\
2088 & Badja Mtn., N.S.W. CSIRO seedlot No. 11861 & $2.6 \mathrm{~b}$ \\
\hline
\end{tabular}

TABLE 13-Eucalyptus dalrympleana provenance differences at Kahuiti

\begin{tabular}{cllll}
\hline Accession & \multicolumn{1}{c}{ Provenance } & $\begin{array}{c}\text { Vigour } \\
(0-5)\end{array}$ & $\begin{array}{c}\text { Crown } \\
\text { form } \\
(0-5)\end{array}$ & $\begin{array}{c}\text { Utility } \\
\text { rating } \\
(0-5)\end{array}$ \\
\hline 1969 & $\begin{array}{l}\text { Mullion Creek, N.S.W. 915 m a.s.l. } \\
\text { FRI DA/1 HO/73/794 FRES77/394 }\end{array}$ & $2.8 \mathrm{a}$ & $3.6 \mathrm{a}$ & $1.4 \mathrm{a}$ \\
1971 & $\begin{array}{l}\text { Brindabella, N.S.W. 1200 m a.s.l. NZ FRI } \\
\text { DA/3 77/2109 CSIRO seedlot No. S12190 } \\
1130\end{array}$ & $2.6 \mathrm{ab}$ & $3.7 \mathrm{a}$ & $1.2 \mathrm{ab}$ \\
& $\begin{array}{l}\text { South Bull Head, A.C.T. 760 m a.s.1. } \\
\text { CSIRO seedlot No. 7890 }\end{array}$ & $2.2 \mathrm{ab}$ & $3.2 \mathrm{ab}$ & $0.6 \mathrm{ab}$ \\
1129 & $\begin{array}{l}\text { Steppes, Tasmania. 900 m a.s.l. } \\
\text { CSIRO seedlot No. 12517 }\end{array}$ & $2.1 \mathrm{ab}$ & $3.2 \mathrm{ab}$ & $0.2 \mathrm{ab}$ \\
& $\begin{array}{l}\text { Wihareja, Tasmania. 850 m a.s.1. NZ FRI } \\
\text { DA/2 FRI77/2108 CSIRO seedlot No. S11721 }\end{array}$ & $1.6 \mathrm{~b}$ & $2.7 \mathrm{~b}$ & $0.0 \mathrm{~b}$ \\
\hline
\end{tabular}

TABLE 14-Eucalyptus fastigata provenance differences at Kahuiti

\begin{tabular}{|c|c|c|}
\hline Accession & Provenance & $\begin{array}{l}\text { Utility rating } \\
(0-5)\end{array}$ \\
\hline 1978 & Oakura, Taranaki. NZ FRI FA/110 FRI78/2214 & $3.7 \mathrm{a}$ \\
\hline 1979 & $\begin{array}{l}\text { Stewarts Brook State Forest, Barrington Tops, N.S.W. } \\
\text { NZ FRI FA/122 FRI78/2284 HO/0/78/46 }\end{array}$ & $2.2 \mathrm{ab}$ \\
\hline 2426 & $\begin{array}{l}\text { Errinundra Plateau, Bendoc, Victoria. } 1120 \mathrm{~m} \text { a.s.l. } \\
\text { NZ FRI FA/11 HO/0/77/21 }\end{array}$ & $2.2 \mathrm{ab}$ \\
\hline 2427 & $\begin{array}{l}\text { Robertson, N.S.W. } 670 \mathrm{~m} \text { a.s.l. } \\
\text { NZ FRI FA/113 HO/0/78/20 }\end{array}$ & $1.1 \mathrm{~b}$ \\
\hline
\end{tabular}


seedlots on two central North island sites and at Longwood Forest. No convincing ranking of $E$. nitens provenances was apparent at Kahuiti.

Fourteen of 16 Eucalyptus species in a species selection trial at Longwood Forest were also represented in the Wairarapa trials. In an assessment of the Longwood trial 6 years after planting (Wilcox et al. 1985), performance of four provenances of $E$. sieberi was disappointing with poor form and branching and frost damage. By contrast, performance and form of E. sieberi was good in the Wairarapa trials, although survival at Kahuiti was patchy. At Longwood the Nerrigundah, Fingal, and Newnes provenances ranked well above an apparently inferior Currarong (N.S.W.) provenance. Neither the better nor the worse performing E. sieberi provenances at Pakaraka (Table 6) were present at Longwood.

Two of the nine $E$. obliqua provenances at Longwood were also represented in the Wairarapa trials, along with (different) seedlots from Mawbanna. The ranking of these for growth was essentially the same in both districts, with Mawbanna at or near the top, Nietta towards the middle, and Powelltown towards the bottom (Table 8), although there were two more inferior provenances (Millicent, S.A., and Bodalla, N.S.W.) at Longwood.

Three of the eight $E$. viminalis provenances at Longwood were in common with the Wairarapa trials. Whereas the Bruthen provenance ranked best for vigour at Pakaraka (Table 11) and (non-significantly) for growth, the Kingston provenance made best growth at Longwood. Across all parameters at Pakaraka there was little to choose between these two or the Cygnet provenance, but the Rotorua provenance performed worst.

The three E. dalrympleana provenances at Longwood-Mullion Creek, Brindabella, and Wihareja-ranked in the same order for growth there as for vigour and utility rating at Kahuiti (Table 13).

Little differentiation was evident between the four $E$. fastigata provenances at Pakaraka, the ranges of differences in provenance means being $0.8 \mathrm{~m}$ for height and $2.0 \mathrm{~cm}$ for best $\mathrm{dbh}$. The Oakura provenance ranked best for all the parameters combined at Kahuiti, and was significantly better than the Robertson provenance for the utility rating (Table 14). In a trial of $126 \mathrm{E}$. fastigata provenances planted on two central North Island sites and assessed a year after planting, the early rapid growth of Oakura provenance featured with that of a Hunterville provenance, although both suffered frost damage and became severely forked (Wilcox 1982b). The inference that it would perform to advantage on a milder site (Wilcox $1982 b$ ) was thus confirmed at Kahuiti. Of note in passing is that the Errinundra Plateau and Robertson provenances proved even less frost-hardy in a controlled-environment frost test, and the Barrington Tops provenance was the most frost-tolerant (Wilcox et al. 1980).

Provenance performance rankings at Pakaraka and Kahuiti generally supported rankings observed in Forest Research Institute provenance selection trials, differences being attributable to frost on the FRI sites or to geographic location as with E. regnans at Longwood Forest.

\section{APPLICATION OF RESULTS}

Parakaka is clearly the more suitable of the two sites for eucalypts. There eucalypts are an appropriate choice for soil conservation plantings or agroforestry. Grazing animals can be re-admitted 3 years after planting (or, with due caution, possibly even earlier). There may 
be advantages in planting a mixture of species to help minimise the effects of insect pests and to allow for variations in site tolerances. Closer initial planting than required for soil conservation alone will allow for some differential survival and medium-term harvesting (or possibly coppicing) of excess trees for timber or firewood crops. A modest amount of silviculture is worthwhile even for soil conservation alone (and essential for production forestry), as many of the trees of inherently good shape at Pakaraka would have been greatly improved by some form pruning.

The implication in the (low) mean utility rating for the upper grouping at Kahuiti is clear. Eucalypts grow well on some parts of the site, while lower parts of the site would be well suited to poplars and willows. However, given the variability of the site and the continuing and more deep-seated erosion, widely spaced trees to keep the site in pastoral use are not the appropriate option. Plantation planting is required to protect adjacent pasturage, and production forestry is the obvious economic land use.

Recommended larger-growing eucalypts for widely spaced soil conservation plantings on eastern Wairarapa hill country are E. regnans*, E. obliqua, E. fastigata, E. botryoides, $E$. botryoides x saligna, $E$. nitens, $E$. fraxinoides, $E$. sieberi, $E$. delegatensis*, $E$. viminalis, E. globoidea, E. smithii, and E. oreades. Recommended smaller stature species are E. amygdalina, E. cordata, E. brookeriana, E. radiata, E. cladocalyx, E. pulchella, $E$. agglomerata, and E. nitida; and for a moister disturbed site like Kahuiti, E. kitsoniana Maid. and E. dalrympleana. In the longer term, the larger-growing trees are more likely to suit conservation/production woodlots and agroforestry regimes, and the smaller trees more single-purpose, widely spaced, hillside stabilisation plantings. Acacia dealbata and A. melanoxylon rank with eucalypts as woodlot options, the former on better-drained sites and the latter on moister sites and more disturbed soils.

\section{ACKNOWLEDGMENTS}

R.L. Hathaway and M. King were responsible for establishing the trials and carrying out the earlier assessments, assisted by S. Blakemore, P. Cameron, M. Vine, C. Stace, and P. Yalden. C. Stace, M. Evans, and L. Muir assisted with the latest assessment. The manuscript for the latter was helpfully reviewed by P. Cannon, J. Miller, M.D.Wilcox, J.S. Sheppard, and A.N. Gilchrist.

\section{REFERENCES}

CHIPPENDALE, G.K. 1988: Myrtaceae - Eucalyptus, Angophora.In "Flora of Australia", Vol. 19. $543 \mathrm{p}$.

EYLES, G.O. 1983: The distribution and severity of present soil erosion in New Zealand. N.Z. Geographer 39(1): 1-8. (Reprinted as pp.281-90 in van Kraayenoord, C.W.S.; Hathaway, R.L. (Ed.) 1986: "Plant Materials Handbook for Soil Conservation. Vol. 1: Principles and Practices." Water and Soil Miscellaneous Publication No. 93).

FOREST RESEARCH INSTITUTE 1990: Success with biological control of the eucalyptus tortoise beetle, Paropsis charybdis. Ministry of Forestry, Forest Research Institute, What's New in Forest Research No. 184.

* This recommendation, based on the Wairarapa trials, must be qualified by incidence of a pathological syndrome which has resulted in severe mortality of $E$. regnans and $E$. delegatensis on some New Zealand sites subject to warm wet summers (M. Kay, FRI, pers. comm.). 
HATHAWAY, R.L. 1986a: Plant materials for slope stabilisation. Pp. 39-47 in van Kraayenoord, C.W.S.; Hathaway, R.L. (Ed.): "Plant Materials Handbook for Soil Conservation. Vol. 1: Principles and Practices." Water and Soil Miscellaneous Publication No. 93.

1986b: Plant materials for gully control. Pp. 49-56 in van Kraayenoord, C.W.S.; Hathaway, R.L. (Ed.): "Plant Materials Handbook for Soil Conservation. Vol. 1: Principles and Practices." Water and Soil Miscellaneous Publication No. 93.

HATHAWAY, R.L.; KING, M. 1986: Selection of Eucalyptus species for soil conservation planting in seasonally dry hill country. New Zealand Journal of Forestry Science 16(2): 142-51.

HATHAWAY, R.L.; SHEPPARD, J.S. 1986: Management and uses of Eucalyptus spp. (eucalypts). Pp. 49-69 in van Kraayenoord, C.W.S.; Hathaway, R.L. (Ed.): "Plant Materials Handbook for Soil Conservation. Vol. 2: Introduced Plants." Water and Soil Miscellaneous Publication No. 94.

JOHNSTON, G.R.; WILCOX, M.D. 1989: Eucalyptus species trials on pumiceland. New Zealand Forestry 34(1): 24-7.

KING, J.N.; WILCOX, M.D. 1988: Family tests as a basis for the genetic improvement of Eucalyptus nitens in New Zealand. New Zealand Journal of Forestry Science 18(3): 253-66.

METEOROLOGICAL SERVICE 1986: Summaries of water balance data for New Zealand Stations. N.Z. Meteorological Service Miscellaneous Publication 189. 102 p.

THOMPSON, C.S. 1982: The weather and climate of the Wairarapa region. N.Z. Meteorological Service Miscellaneous Publication 115(11). 60 p.

WILCOX, M.D. 1980: Genetic improvement of eucalypts in New Zealand. New Zealand Journal of Forestry Science 10(2): 343-59.

1982a: Preliminary selection of suitable provenances of Eucalyptus regnans for New Zealand. New Zealand Journal of Forestry Science 12(3): 468-79.

1982b: Genetic variation in frost tolerance, early height growth, and incidence of forking among and within provenances of Eucalyptus fastigata. New Zealand Journal of Forestry Science 12(3): 510-24.

WILCOX, M.D.; THULIN, I.J. 1979: Growth of Eucalyptus regnans in a plot at Rotorua. New Zealand Journal of Forestry Science 9(2): 166-9.

WILCOX, M.D.; ROOK, D.A.; HOLDEN, D.G. 1980: Provenance variation in frost resistance of Eucalyptus fastigata Deane and Maid. Paper presented to IUFRO Symposium and Workshop on "Genetic Improvement and Productivity of Fast-Growing Tree Species", Aguas de Sao Paulo, Brazil, August 1980.

WILCOX, M.D.; MILLER, J.T.; WILLIAMS, I.M.; GOULD, D.W. 1985: Eucalyptus species trials in Longwood Forest, Southland. New Zealand Forest Service, FRI Bulletin No. $95.21 \mathrm{p}$. 


\section{APPENDIX}

\section{SUPPLEMENTARY OBSERVATIONS ON INDIVIDUAL SPECIES}

(series classification within the Eucalyptus genus according to Chippendale 1988)

Acacia dealbata (syn. Racosperma dealbatum ) - upper grouping at Pakaraka, middle grouping at Kahuiti, good vigour at Pakaraka but heavily branched and spreading crown suppresses pasture (typically for wattles in general), more a candidate for more closely spaced plantings in areas retired from grazing.

A. longifolia var. sophorae (syn. $R$. sophorae) - lower grouping at both sites, a multistemmed spreading shrub which grew well but did not conform to the selection criteria for widely spaced trees.

A. mearnsii (syn. R. mearnsii) - lower grouping at Pakaraka, middle at Kahuiti, poor form, subject to Uromycladium gall rust.

A. melanoxylon (syn. R. melanoxylon) - middle grouping at Pakaraka, upper at Kahuiti, tended to be outperformed by eucalypts at Pakaraka, more consistent than most eucalypts at Kahuiti, slow compared with eucalypts, profusely branched and needs silviculture when grown at wide spacings, more suited to production/conservation woodlots.

Casuarina cunninghamiana, C. glauca - vigour generally poor but some trees had good form.

Eucalyptus agglomerata - upper grouping at Pakaraka, lower at Kahuiti, among the smaller trees in the upper grouping Pakaraka (but can also be a tall forest tree in its natural habitat), stringybark group, series Pachyphloiea.

E. amygdalina - upper grouping at both sites, conformed to selection criteria well, ultimately smaller stature tree, peppermint group, series Radiatae.

E. botryoides - upper grouping at both sites, best individual species at Kahuiti, somewhat heavily branched at wide spacings, adopted a rounded spreading habit where exposed to the wind at Pakaraka, suited to production/conservation woodlots, eastern blue gum group, series Transversae.

E. botryoides $\times$ saligna - upper grouping at both sites, of better form than $E$. botryoides but a little less vigorous at Kahuiti, hybrid of eastern blue gum group species, series Transversae.

E. brookeriana - upper groupings at both sites despite susceptibility to insect damage, scored well for growth habit, regarded as ultimately of smaller stature (but can become a tall forest tree in its natural habitat), swamp gum group, series Foveolatae.

E. cinerea - lower grouping at Pakaraka, middle at Kahuiti, ranked among first 10 at Kahuiti for growth, scored poorly at both sites for form, just average for vigour, ultimately smaller stature tree, gum group, series Viminales.

E. cladocalyx - upper grouping at Pakaraka, unranked at Kahuiti, good growth and form at Pakaraka, poor survival at Kahuiti, "sugar gum", series Microcorythae.

E. cordata - upper grouping at both sites, near top at Kahuiti, expected to ultimately be a smaller stature tree (but currently not far behind the largest at Kahuiti), gum group, series Viminales. 
E. dalrympleana - unranked at Pakaraka, upper grouping at Kahuiti, poor survival at Pakaraka, very good survival at Kahuiti, slow, small stature tree (but can become a tall forest tree in its natural habitat), not well rated for form and insect resistance, could be planted at closer spacings than the other upper grouping species without forming a closed canopy (for a long time) and so possibly an option where an eroding site needs to be retired from grazing and closely planted with trees but an ultimately re-opening for grazing is a possibility, gum group, series Viminales.

E. delegatensis - upper grouping at Pakaraka, middle at Kahuiti, was not in the top 15 species at either site at 5 years but near the top of the upper grouping at Pakaraka after 12 years, well ranked for form, ash group, series Eucalyptus.

E. fastigata - near top of upper grouping for both sites, rather heavily branched at Pakaraka, form better at Kahuiti where as one of the more successful species it ended up at closer spacings, ash group, series Regnantes.

E. fraxinoides - upper grouping at both sites, performance downgraded by a tendency to heavy branching, failure at Kahuiti on the more poorly drained and disturbed parts of the site, ash group, series Fraxinales.

E. globoidea - upper grouping at Pakaraka, lower at Kahuiti, candidate for agroforestry or woodlot (naturally durable) timber production, stringybark group, series Pachyphloiae.

E. johnstonii (syn. E. vernicosa ssp. johnstonii) - lower grouping at Pakaraka, middle at Kahuiti, amongst the smaller trees in the trials (but a tall forest tree in its natural habitat), columnar habit suits both windbreaks and widely spaced planting, too susceptible to insect damage for the Wairarapa region, gum group, series Viminales.

E. kitsoniana - middle grouping at Pakaraka, upper at Kahuiti, very good survival and consistent performance at Kahuiti despite average vigour and below average form, ultimately a smaller stature tree, gum group, series Viminales.

E. kybeanensis - middle grouping at Pakaraka, unranked at Kahuiti, good vigour and insect resistance, reasonable form for widely spaced plantings, smaller stature tree, ash group, series Fraxinales.

E. macrorhyncha - middle grouping at Pakaraka, lower at Kahuiti, good vigour, reasonable habit for widely spaced plantings, ultimately a smaller stature tree, stringybark group, series Pachyphloiae.

E. maculata - middle grouping at Pakaraka, unranked at Kahuiti, conformed to selection criteria reasonably well at Pakaraka, gum bleeding from branch scars tends to be unsightly, "spotted gum", series Maculatae.

E. muelleriana - unranked at both sites, formerly amongst top 15 at Pakaraka at 5 years, poor survival but survivors a little above average for growth, good for vigour, average for form, more of a species for (naturally durable) timber production woodlots with more attention to siting and establishment, stringybark group, series Pachyphloiae.

E. nitens - upper grouping at both sites, vigour good despite insect predation of adult foliage, form rather more spreading and heavily branched than desirable for widely spaced plantings, a candidate for interplanting with slower-growing species for early effectiveness and later removal in a production thinning, southern blue gum group, series Viminales. 
E. nitida - upper grouping at Pakaraka, unranked at Kahuiti, conformed well to selection criteria at Pakaraka, ultimately smaller stature tree, peppermint group, series Radiatae.

E. obliqua - upper grouping at both sites, good consistency for growth over both sites, more heavily branched than ideal for widely spaced trees, ash group, series Eucalyptus.

E. oreades - upper grouping at Pakaraka, unranked at Kahuiti, best on well-drained undisturbed soils low on the slope, ash group, series Eucalyptus.

E. ovata - middle grouping at both sites, performance a little above average but downgraded by susceptibility to insect damage, swamp gum group, series Foveolatae.

E. pulchella - upper grouping at Pakaraka, unranked at Kahuiti, conformed to selection criteria well at Pakaraka, formerly in top 15 at both sites after 5 years, subject to wind damage apparently through trees in open stands becoming multileadered and then the leaders becoming major upright branches as the canopy closed in, smaller stature tree, peppermint group, series Radiatae.

E. radiata - upper grouping at Pakaraka, middle at Kahuiti, conformed to selection criteria well (but some provenances become spreading with age), some provenances ultimately smaller stature trees, peppermint group, series Radiatae.

E. regnans - upper grouping at both sites, best species for the selection criteria overall, odd trees declining and dying on the wetter parts of Kahuiti, ash group, series Regnantes.

E. risdonii - middle grouping at Pakaraka, lower at Kahuiti, very good vigour at Pakaraka, reasonable form for widely spaced plantings, smaller stature tree, peppermint group, series Radiatae.

E. smithii - upper grouping at Pakaraka, lower at Kahuiti, good growth and vigour at Pakaraka, poor form and insect resistance, notable for vigorous coppice regrowth after thinning, gum group, series Viminales.

E. sieberi - upper grouping at Pakaraka, middle at Kahuiti, growth and form good, survival patchy at Kahuiti, ash group, series Psathyroxyla.

E. stellulata - middle grouping at both sites, formerly in top 15 at Pakaraka after 5 years, form downgraded by crooked stems, smaller stature tree, ash group, series Longitudinales.

E. viminalis - upper grouping at Pakaraka, middle at Kahuiti, good growth at Pakaraka, formerly in top 15 at Kahuiti after 5 years, form and insect resistance variable and often poor, a candidate for closer spacings for early effectiveness and later harvesting/ culling rather than a long-term component of widely spaced plantings. 